\title{
中国心理学前沿
}

\section{初中生自我效能感与考试 焦虑的关系研究}

\author{
汪振国 * 余欣欣 \\ 广西师范大学教育学部, 认知神经科学与应用心理重点实验室, 桂林, 541000
}

邮箱: 644878113@qq.com

摘 要: 目的: 研究吉安市初中生自我效能感和考试焦虑水平的特点及其潜在 关系, 为降低考试焦虑提供科学依据。方法: 选取吉安市 4 所中学 1112 名初中 生作为研究对象, 使用 Sarason 考试焦虑焦虑测验 ( TAS ) 和自我效能感 (GSES) 两个自评量表进行测评, 使用 SPSS 22.0 中文版软件对结果进行统计。结果: (1) 吉安市初中生自我效能感和考试焦虑在性别上存在显著差异 $(t=3.75, p<0.01$; $t=-2.34, p<0.05)$; 2)吉安市初中生自我效能感和考试焦虑在年级上差异不 显著 ( $p>0.05)$; (3)吉安市初中生自我效能感在出生地上存在显著差异 $(t=-3.64$, $p<0.01)$; (4)吉安市初中生自我效能感和考试焦虑呈显著的负相关 $(r=-0.30$, $p<0.001$ )。结论：(1)初中生的自我效能感与性别、生源地有关; (2)自我效能感 影响考试焦虑水平。

关键词: 初中生; 自我效能感; 考试焦虑

投稿日期：2018-09-12；录用日期：2019-03-23；发表日期：2019-06-03

文章引用: 汪振国, 余欣欣. 初中生自我效能感与考试焦虑的关系研究 [J]. 中国心理学前沿, 2019, 1 ( 4 ): 233-250. 


\title{
A Study on Self Efficacy and Test Anxiety of Junior High School Students
}

\author{
Zhenguo Wang* Xinxin Yu
}

Key Laboratory of Cognitive neuroscience and applied Psychology, Guangxi Normal Universit, Guilin 541000

Abstract: Purpose: The study aimed to explore the relation between self efficacy and test anxiety for junior high school students in Ji'an.Participants are 1263 junior high school students in Ji'an. Tools: Sarason anxiety and anxiety test (TAS) and self efficacy scale (GSES) were used in this study. Results: (1) The differences of gender were significant on self-efficacy and test anxiety for junior high school students in Ji'an $(t=3.75, p<0.01 ; t=-2.34, p<0.05) ;(2)$ There was no grade differences on selfefficacy and text anxiety for junior high school students in Ji'an $(p>0.05)$; (3) The differences of region were obvious on self-efficacy for junior high school students in Ji'an $(t=-3.64, p<0.01)$; (4) Two variables were notable related.Self-efficacy correlated to text anxiety $(r=-0.30, p<0.001)$. Conclusions: (1) gender value has an effect on self-efficacy and test anxiety of junior high school students; (2) The birthplace has an impact on the quality of children.

Key words: Junior high school students; Self-efficacy; Test anxiety

Received: 2019-20-12; Accepted: 2019-03-23; Published: 2019-06-03

Copyright (C 2019 by author(s) and SciScan Publishing Limited.

This work is licensed under a Creative Commons Attribution 4.0 International License. https://creativecommons.org/licenses/by/4.0/ 


\section{1 引言}

中国是考试的发祥地，早在隋朝，为了适应封建经济和政治的发展变化， 隋炀帝开创了科举制, 这一制度在唐朝得到完善, 并沿用至今。考试为知识份 子开辟了一条完成自我抱负的伟大航道，也最大限度地节约了政府选择人才的 资源, 可谓一石二鸟。但是随着考试的出现, 考试焦虑这一体验也从未消失。

一直以来, 教育界和心理学界对于初中生学业成绩的关注从未减弱, 影响学生 学业成绩的因素在教育中是一个重要的问题, 国外的研究发现, 自我效能感和 考试焦虑对于学业的表现具有潜在影响 $[1]$ 。在中国, 初中学生中存在着一个 特殊的群体, 就是面临中考的九年级学生, 研究者往往对该群体深感兴趣, 使 其成为研究的主要对象。但是, 九年级学生的考试焦虑主要由于中考临近这个 应激事件而产生，考试焦虑伴随着中考临近这个额外变量而得到加强，将初中 生群体作为研究对象而不考虑中考临近这一额外因素是不准确的, 所以本研究 仅选取七、八两个年级的学生做为研究对象, 从而较好地排除了临近中考这一 因素的影响。

考试焦虑 ( Text Anxiety) 是一种复杂情绪体验, 针对考试焦虑的研究有很 多, 但这一概念目前依旧没得到统一的界定。在国内, 学习测验是检验学习成 果的最好方式，对于初中生来说，检验自己学习最有效的手段就是学业考试。 考生在进行学业测验时, 由于过度担忧测验结果而使得自身能力没有真正得以 表现出来，这种在考场上或面临考试而产生的消极感受称为考试焦虑 [2]。 Adewuyi, Taiwo 和 Olley 解释说考试焦虑主要是由担忧和紧张两种元素所构成 [ 3 ]。 一项研究以北京初中生为例, 对北京市 622 名中学生进行匿名网络调查。结果 发现, 中学生焦虑水平的平均得分为 $(36.1 \pm 9.7)$ 分, 其中, 处于镇定水平的 学生占总体的 $29.4 \%$, 轻度焦虑占 $47.6 \%$, 中度焦虑占 $19.8 \%$, 重度焦虑占 $3.2 \%$ [ 4 。 以上研究可以知道, 大约有 $70.6 \%$ 的初中生在考试焦虑水平上呈现出焦虑状态。 初中生在学习教育的过程中, 考试测验无形中也成为了学生焦虑和担忧的源泉。 
实际上, 学生们面对考试时产生焦虑是很自然的事情, 任何人可能都会体验到 考试焦虑，对于初中生来说，最容易产生焦虑的考试莫过于中考。目前有学者 认为, 考试焦虑不是在单独因素影响下发生作用, 而是在某种应激情景下, 在 各种因素的共同影响之下共同发挥作用，对考试结果焦虑主要是以逃避、防御 等消极情绪反应为手段而成为其最主要的特点。笔者采用的是田宝对考试焦虑 的界定，即考试焦虑是由考试引起的焦虑 [5]。

考试焦虑有两种: 第一种是在学业测验过程中感受到的消极情感体验; 第 二种是在学业测试之前所感受到的消极情感体验。当考生体验到考试焦虑这种 消极体验时, 不仅在生理上会有所反应, 而且在心理上也会出现不同的反应 $[6]$ 。 例如, 在生理方面会出现四肢无力、心跳不规律等症状; 在心理上产生难受、 习得性无助、烦扰等情绪体验, 随着考试焦虑水平不断增加, 其生理反应也更 为严重, 如视力下降、发烧、呕吐; 在心理方面, 则会出现精神无法集中、思 维处于呆滞状态，无法深人思考，无法进行记忆等问题，更严重的可能会导致 人眩晕甚至晕厥, 心理上产生考试恐惧症, 逃避学校等极端情绪。

造成考试焦虑的原因有许多，各种原因都在发挥着自身独特的作用，其中 自我效能感是不可忽视的因素之一。各种因素的交互作用之下造成了考试焦虑。 焦虑可分为内源性成分和外源性成分。内源性成分包括遗传成分、生理成熟度、 个体认知水平、知识的储备水平和考试经验等; 外源成分包括家庭成分、学校 成分和社会影响等。在外源成分和内源成分的共同影响下，考试焦虑便产生。

自我效能感最早由美国心理学家 Bandura 提出, 并且是他的社会认知理论中 的核心概念。自我效能感指人们自身对于能否有能力完成某项工作行为的自信 程度 $[7]$ 。

郑希付 (2003) 认为, 考试焦虑与自我效能感有关 [8]。自我效能感能通 过影响个体注意的对象和对困难的态度来改变焦虑水平。当判断自己是无效能 感或者低效能感的时候，这类学生在应对相同的问题时，一般只会去思考自己 个人的缺点和认识上的潜在难题, 把问题难度在个人的主观思维里不断加大, 这种认识直接导致了他们不注重怎么去更好的行动, 而只关心个人最终的结果, 从而在内心中会产生巨大的焦虑感，这样的焦虑感直接损害了自身能力的发挥。 相反, 高自我效能感的学生, 不像低自我效能感的人在面临困难时, 只会考虑 自身的不足，降低自我能力，他们会把自身的注意力集中到应对环境的要求和 
困难的解决之上，这样就集中了他们解决问题的能力，提高了战胜困难的决心， 在不经意间提升了他们对自身的期望。班杜拉认为，自我效能感在人类动机中 是占据核心地位的。

近期各种研究也显示出了自我效能和考试焦虑之间呈负相关，Schwarzer 和 Ierusalem ( 1992 ) 的研究中发现自我效能感和考试焦虑之间的关系为 $-0.3 \sim-0.66$ [9]。在赵显和许艳君的研究中也发现, 这两者也呈负相关, 当考试焦虑分数高, 自我效能感水平就会相对较弱; 相反，当考试焦分数低，自我效能感水平就会 较强。

综上所述，可以发现，自我效能感对考试焦虑有影响。基于此，本研究做 出下假设：在吉安市初中生中，自我效能感与考试焦虑呈负相关。

\section{2 对象与方法}

\section{1 研究对象}

本研究随机抽取吉安市城北中学、吉安五中、吉安思源实验学校和吉安二 中 24 个班，共计 1263 名在校学生七、八年级学生，笁查后有效问卷为 1112 份, 其中被试详细信息表 1 所示。

表 1 被试基本信息

\begin{tabular}{cccc}
\hline 项目 & 类别 & 人数 & 百分比 $(\%)$ \\
\hline \multirow{2}{*}{ 性别 } & 男 & 596 & 53.6 \\
& 女 & 516 & 46.4 \\
生源地 & 农村 & 299 & 26.3 \\
& 城镇 & 813 & 73.7 \\
年级 & 七年级 & 607 & 54.6 \\
& 八年级 & 505 & 45.4 \\
\hline
\end{tabular}

\section{2 研究工具}

2.2.1一般自 我效 能 感量表 (General Self-efficacy Scale, GSES )

Schwarzer 和他的同事于 1981 年编制了一般自我效能感量表（General Self-efficacy Scale，GSES）。GSES 开始有 20 个项目，后来改进为 10 个项目。目前 GSES 已被翻 译成至少 25 种语言，并在国际上广泛使用。中文版的一般自我效能感量表在各项 
研究中已经被证实拥有较好的信度和预测效度。一般自我效能感量表共有 10 个 题目，这 10 个题目不仅涉及个体遇到挫折时的自信心水平，而且还包括遭遇困 难时的自信心水平。一般自我效能感量表采用 4 点计分法，总分越高，自我效 能感越高。

\subsection{2 考试焦虑量表 ( Text Anxiety Scale, TAS )}

Sarason 考试焦虑量表是用来考察考试焦虑程度的测验量表，由 37 道题目组 成, 内部一致性为 0.87 , 重测信度为 0.59 , 验证性因素显示量表有较好的结构 [ 10 。 量表中每个项目均有 “是” “否” 两个选项，依据量表进行统计分数，总分越低， 考试焦虑水平也就会越低。

\section{3 统计学处理}

所有数据通过 $\operatorname{spss} 22.0$ 中文版软件进行统计分析。主要采取独立样本 $t$ 检验, 配对样本 $t$ 检验及相关分析。

\section{3 结果与分析}

\section{1 被试自我效能感和考试焦虑水平}

根据 GSES 和 TAS 量表分数划分，自我效能感低的被试为 4 人，占 $0.3 \%$; 自我效能感较低的被试有 212 人，占 $19.1 \%$; 自我效能感较高的被试有 737 人， 占 $66.3 \%$; 自我效能感高的被试有 159 人，占 $14.3 \%$ 。在考试焦虑水平上，低焦 虑被试有 404 人，占 $36.3 \%$; 中等焦虑被试有 427 人，占 $38.4 \%$; 高焦虑被试有 281 人，占 $25.3 \%$ 。样本具体信息如表 2 所示。

表 2 被试自我效能感和考试焦虑水平分布

\begin{tabular}{cccc}
\hline 项目 & 水平 & 人数 & 百分比 $(\%)$ \\
\hline & 低 & 4 & 0.3 \\
自我效能感 & 较低 & 212 & 19.1 \\
& 较高 & 737 & 66.3 \\
& 高 & 159 & 14.3 \\
& 低 & 404 & 36.3 \\
考试焦虑 & 中等 & 427 & 38.4 \\
& 高 & 281 & 25.3 \\
\hline
\end{tabular}




\section{2 自我效能感与考试焦虑在人口学意义上的差异检验}

\subsection{1 考试焦虑、自我效能感在性别上的差异检验}

对自我效能感和考试焦虑的性别差异进行独立样本 $t$ 检验。自我效能感在性 别上具有十分显著的差异，且表现为男生的自我效能感高于女生; 考试焦虑在 性别上不存在显著差异。样本具体信息如表 3 所示。

\section{表 3 自我效能感和考试焦虑在性别、生源地和年级上的差异}

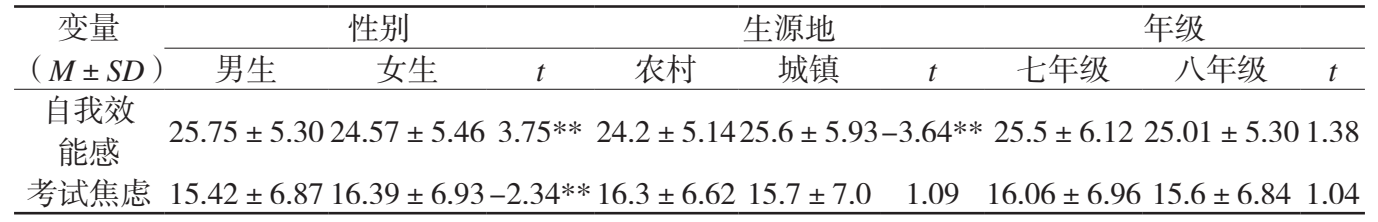

注: *代表 $p<0.05, * *$ 代表 $p<0.01$ 。

3.2 .2 考试焦虑、自我效能感在生源地上的方差检验

如表所示, 自我效能感在生源地上具有十分显著的差异, 且表现为城镇学 生自我效能感高于农村学生; 考试焦虑在生源地上不存在显著差异。

\subsection{3 自我效能感和考试焦虑在年级上的差异检验}

如表所示，自我效能感和考试焦虑在年级上均不存在显著差异。

3.2 .4 考试焦虑水平在自我效能感高分组和低分组上的差异检验

选取自我效能感高分组和低分组，高分组和低分组是按照排名的前 $27 \%$ 和 后 $27 \%$ 进行定义，采取配对样本 $t$ 检验，自我效能感高、低分组对应的考试焦 虑水平具有十分显著的差异。样本具体信息如表 4 所示。

表 4 考试焦虑在自我效能感高分组和低分组的差异检验 $(N=341)$

\begin{tabular}{cccc}
\hline 组别 & 高分组 $(M \pm S D)$ & 低分组 $(M \pm S D)$ & $t$ \\
\hline 自我效能感 & $-4.99 \pm 9.78$ & $-4.99 \pm 9.78$ & $-9.43^{* *}$ \\
\hline
\end{tabular}

注: **代表 $p<0.01$ 。

3.2.5 吉安市初中生自我效能感和考试焦虑之间的关系

对自我效能感和考试焦虑进行相关分析，自我效能感和考试焦虑的 pearson 相关为 -0.3 。样本具体信息如表 5 所示。 
表 5 自我效能感和考试焦虑的 pearson 相关

\begin{tabular}{cc}
\hline & 自我效能感 \\
\hline 考试焦虑 & $-0.3^{* * * *}$ \\
\hline
\end{tabular}

注: ***代表 $p<0.001$ 。

\section{4 讨论}

\section{1 自我效能感和考试焦虑与性别的关系}

如表 3 所示, 吉安市初中生的自我效能感和考试焦虑在性别上具有显著差异, 与刘朝霞 (2015) 研究结果不完全一致 [11]。在当今社会中, 男生和女生面 临着相同的学习任务, 在对于成绩的要求上已经完成了 “男女平等” 的对待模式。 因此社会对于初中生，无论从能力方面还是学习方面，都有着相同的期待。

首先，吉安市初中生自我效能感在性别上有着显著差异，男生自我效能感 高于女生。初中生正处于青春期, 在这个特殊的阶段的学生极易受到社会因素、 学校因素和家庭因素等多方面的影响, 特别是社会文化因素和家庭教育因素的 影响。男性在中国传统的教育中要求是勇敢、坚韧不拔、勇于挑战的，男性的 自我效能感的会在中国传统文化的熏陶下逐渐的成长起来。男性被要求完成各 种富有挑战性的任务，并被给予丰厚的物质和精神奖励，在不断增加自身实力 的同时自我效能感也在悄无声息的增加。自从进人父系社会, 男性的地位就不 断得到巩固, 为了保障男性地位的传承性, 形成了“男尊女卑” 的传承文化, 在 这种文化思潮的引导之下，中国经历了上千年，每个中国人的精神世界都打下了 深刻的烙印, 这一结果在如今文明的今天也难以完全改变。在这种文化之下, 男 性不断增强自身实力, 不断赢得对自身的肯定, 自我效能感的增加也成为必然。 反观在这种文化影响下, 女性被认为是柔弱的, 是需要被保护的, 自身对于自己 的能力本身就不确定, 而且被男性保护也被女性视为荣誉, 这样女性便直接或间 接地失去了许多提升自身能力的机会, 自我效能感从而也增加得缓慢。

在家庭教育方面亦如此。即使到了今天，在幼小的孩子中，文文静静的女 生被认为是有教养、知书达理的, 被人们所喜爱; 而敢于冒险的男生总是被认 为是优秀、今后有所作为的, 亦被人们所接受。在人们接受的同时, 直接发生 
作用的场所就在家庭。父母是孩子的第一任老师，性别认同也是在孩子幼小阶 段在父母教育中完成的。调皮的女生和不爱说话的男生将会受到批评和惩罚， 以至于孩子不得不认同父母，以免遭到惩罚。这样在社会因素和家庭因素的共 同影响下，男、女生的自我效能感存在差异也是可以理解的。

在表 3 中，考试焦虑的性别差异也是显著的，女生考试焦虑显著高于男生， 这和李慧杰、王忆军、张慧等 (2005) 研究结果相同 [12]。女生之所以考试 焦虑会高于男生，自我效能感是不能忽视的因素。由于女生的低自我效能感, 使得她们在日常学习中更容易有挫败感, 对自己不自信经常发生, 从而有着比 男生更高的考试焦虑。

\section{2 自我效能感和考试焦虑与生源地的关系}

在自我效能感方面，生源地为城镇的学生自我效能感高于生源地在农村的 学生。这可能是由于在生源地在农村的学生父母主要来自农村, 这些父母对于 孩子自身的自身效能感方面的教育可能存在先天的缺失，加上自身受到上一辈 教育观念的影响, 对于孩子的心理成长是相对空白的; 而出生于城镇的学生, 父母基本已经在城镇生活，容易接受新的教育理念，关注孩子心理成长，在和 其他孩子的对比中无形也增加了自我效能感方面的教育力度。

\section{3 自我效能感和考试焦虑与年级的关系}

考试焦虑和自我效能感在年级上无显著差异, 这项研究结果和多数研究结 果一致。一般认为随着年级的提高考试焦虑会提高，八年级的学生考试焦虑会 比七年级学生高, 考试焦虑会更加严重, 但实际上, 影响考试焦虑的因素还有 许多, 比如社会文化影响、家庭教育和学校影响等, 年级因素只是其中一个而已。 关于自我效能感和考试焦虑上的差异问题，本研究的结果支持自我效能感和考 试焦虑在年级上无显著差异。七年级和八年级学生不会随着年级的上升而出现 自我效能感和考试焦虑水平的显著变化, 学生拥有自我调节能力, 能够顺利地 调节自身在学习上的各种问题。这也从侧面证明了很多研究得出九年级与七、 八年级考试焦虑具有显著差异是受到临近中考这一额外因素影响的结论。 


\section{4 自我效能感和考试焦虑的关系}

吉安市初中生自我效能感和考试焦虑之间的关系为负相关, 并且差异显著。 本研究的结果和朱奕达（2005）的研究结果基本一致 [13]，即自我效能感水 平越高，考试焦虑水平就会越低，其中表 4 也显示出，自我效能感高分组和低 分组对应的考试焦虑水平存在显著差异, 且自我效能感越高, 考试焦虑水平越低。 当个体相信自身可以完成相应的任务，那么该个体有高自我效能感，否则是低 自我效能感。活动的动机水平由许多因素共同决定，但自我效能感是不可忽视 的重要因素之一。自我效能感水平的高低，可以直接决定个体动机水平状况。

高自我效能感的学生往往在学习中更加积极主动，对自己充满克服各种挑战性 任务和战胜挫折的信心，相信自己不仅有能力，而且可以发挥能力，加之自身 的努力完成考试，有取得好成绩的信心，并且对于考试结果多为内部归因方式， 认为考试好与坏都是自身造成的，所以会更加努力的学习; 相反，低自我效能 感的学生认为自己能力不足, 加之经常失败, 使其获得了 “习得性无助” , 转 而厌学，害怕考试，结果也是难以处理的。

Bandura 认为，自我效能感也会影响新技能的学习和已学会技能的运用，高 自我效能感的学生学习知识快, 而且容易掌握运用; 而低自我效能感的学生, 学习知识慢, 运用能力也不足。

\section{5 研究建议}

研究结果表明, 吉安市初中生自我效能感和考试焦虑存在负相关, 即自我 效能感低的学生, 考试焦虑水平会高。这其实提供给我们一个很好的降低吉安 市初中生考试焦虑的方法，即在某种程度上增加学生的自我效能感，就可以减 轻学生的考试焦虑水平。

\subsection{1 增加学生体验成功的机会}

在家庭方面，学生体验成功的感受最初也在家庭之中，这就要求父母可以 在孩子表现很好的时候给予鼓励，这样做不仅起到了行为上强化的作用，更重 要的是让孩子体会到了自己是有能力的，有价值的，可以负责自己行动。比如 可以将家务分配给孩子, 在孩子完成后给予奖励, 这些奖励可以是物质上的奖励, 比如小礼物，美味的零食; 同时也可以是精神上的奖励，比如一个大大的拥抱， 
一个热吻，一个口头表扬，这些都可以让他们体验到成功的奥妙。有条件的父 母还可以依据孩子的兴趣参加其他课程，比如兴趣班，亲子体验活动等，都是 提升学生自我效能感很好的方法。

在学校方面, 可以开展选修课, 这不仅可以促进学生学习的热情, 更重要 的是给予学生更多的成功体验。我们可以开设书法选修课、音乐选修课、美术 选修课、体育选修课等，学校可以因地制宜地开设具有自己特色并且满足学生 需要的课程, 这样可以有效地降低学生因过分专注应试教育而产生的焦虑消极 体验。

\subsection{2 增加获得间接经验的机会}

替代经验比如观察学习、他人示范、自我模仿等都会影响自我效能感。当 看见一个与自己类似的人在一项任务上成功或失败，自我效能感也能够随之提 高或降低。学校可以经常组织励志讲座和学习活动, 让学生们参与, 让他们知 道现在成功的人也经历过失败, 优秀的人也有不明亮的昨天, 传播自身成长经验, 激励学生勇敢克服困难。

\subsection{3 建立“家校一体网络”机制}

所谓 “家校一体网络” 机制指借助现代网络的便捷的工具，如微信、QQ 等 软件，让学校和家庭起到相互帮扶的作用，一方面，对于考试焦虑的孩子，学 校和家庭需要一致劝说，多多引导，共同帮助其降低或者消除考试带来的不利 情绪，共同帮助孩子成长；另一方面，对于生源地为农村的学生，由于父母对 教育认识的不足, 在这一点上学校可以提供较好的建议, 增强家庭对于学生自 我效能感的培养。

\subsection{4 提升学生情绪管理能力}

通过开展心理健康课、团体心理辅导、心理咨询等多种途径帮助学生理解 自身情绪，管理好自身情绪，做情绪的主人。对于难以调节和控制自己情绪的 学生, 学校和家长应该耐心帮助, 真心关怀, 也可以寻求心理咨询的帮助, 通 过多种方法帮助学生提升自我情绪管理能力。

\subsection{5 正确认识性别对自我效能感影响作用}

根据此次研究，我们可以发现，初中男、女生自我效能感存在显著差异。 这就意味着在家庭、学校以及社会文化的共同影响下，导致了女生自我效能感 
培养的严重不足。在这样的影响下，我们更加重视男生的成长，而 “女汉子” 则是被大家不接受的，诸如此类的强化作用会让女生更倾向于表现出 “柔弱” 的一面。长此以往，女生既缺少必要的成长资源，又被 “训练” 成娇弱的姿态， 极其不利于其健康成长。综上所述，加强女生自我效能感训练是十分重要的。

\section{6 研究不足与展望}

\subsection{1 研究不足}

(1)本研究不足在于割裂了初中七、八年级学生和九年级学生的关系，仅仅 研究七、八两个年级学生之间的自我效能感和考试焦虑之间的关系，对于九年 级的研究没有涉及, 使得整个对于初中生的研究并不完整。笔者开始想通过排 除 “中考” 影响因素影响, 但是 “中考” 因素也会对七、八年级产生相应的作用, 这一点笔者难以控制。2)只简单地分析了自我效能感和考试焦虑之间的性别、 生源地、年级以及它们之间的相关性，没能对影响自我效能感和考试焦虑其他 因素进行深度挖掘。3仅仅讨论了吉安市初中生自我效能感和考试焦虑之间的 关系, 其中最近学习成绩也可能是关键的影响因素之一, 但本研究没有讨论。 (4)研究方法较为单一, 存在很大的局限性, 今后应结合其他研究方法进行深人 的研究。

\subsection{2 研究展望}

(1)本研究发现初中生自我效能感和考试焦虑并不是简单的线性关系，希望 接下来的研究可以挖掘其它影响初中生自我效能感和考试的中介变量; (2)本研 究的被试仅为吉安市初中生, 未来的研究被试的跨区域性显得更加重要且具有 科学性。

\section{5 结论}

本研发现初中生的自我效能感与性别、生源地有关, 并且证实了初中生学 生自我效能感与考试焦虑水平之间呈负相关。

\section{参考文献}

[1] Qudsyi H. Self-efficacy and Anxiety of National Examination among High School 
Students [ J ] . Procedia - Social and Behavioral Sciences, 2016, 217: 268-275.

https://doi.org/10.1016/j.sbspro.2016.02.082

[2] 赵显, 许艳君. 高中生自我效能感与考试焦虑的关系 $[\mathrm{J}]$. 校园心理, 2014(5): 328-330.

[3] Olley T. Inference of examination anxity and self-efficacy on academic performance among secondary school students $[\mathrm{J}]$. ABC psychology, 2012.

[4] 陈晨. 北京市城区中学生考试焦虑及影响因素分析 [ J ] . 中国学校卫生, 2010(11): 1323-1324.

[5] 田宝. 高中生考试焦虑影响考试成绩的模式和对策研究 [D ]. 首都师范大学, 2001.

[6] 汪玉秀. 国内中学生考试焦虑的研究综述 [ J ] . 内肚科技, 2009(2): 187.

[7] Wood R, Bandura A. Scia cognitive theory of organizational management [ J ] . Acad Manage Rev, 1989(14): 361. https://doi.org/10.2307/258173

[8] 郑希付. 关于考试焦虑的认知因素研究 [ J ] . 心理科学, 2003, 26(47): 49. https://doi.org/10.1159/000073489

[9] Schwarzer. Advances in anxiety theory:A cognitive process approach $[\mathrm{J}]$. In Advances in anxiety research, 1992, 7(2): 17.

[10］张建新. 考试焦虑量表 (TAS) 在北京市中学生中的适用性 [ J ] . 中国临床 心理学杂志, 2008(6): 623-624.

[11]刘朝霞.初中生学业自我效能感、考试沉浸体验和考试焦虑的关系研究 $[\mathrm{D}]$. 湖南师范大学, 2015.

[12］李慧杰, 王忆军, 张慧, 等.中学生考试焦虑表现的性别差异 [ J ] . 中国临 床康复, 2005(40): 24-26. https://doi.org/10.1176/pn.40.24.0026

[13]朱奕达. 中国学生自我效能感与考试焦虑关系的 Meta 分析 [ J ] . 中国学 校卫生, 2015(4): 527-531. 


\section{附件一：}

请在填写量表前完成下列基本信息，以下全部信息将被严格保密！

年 龄:

性 别:

生源地: (农村 / 城市)

最近一次期末成绩综合排名:

父母是否离婚: (是/否)

家庭成员人数: 人

家庭经济情况： （贫穷/一般/富裕）

所在学校和年级

\section{Sarason 考试焦虑量表（TAS）}

指导语：请仔细阅读每一道题，并根据自己实际情况进行作答。每一道题 都有两种答案 ( A. 是 B. 否), 请从两种答案中选择一个适合你的, 在答题过程 中不得漏题, 在同一题上不得勘酌太多时间, 根据看完题后的第一反应进行作答。 有些题目可能与你不符或你从未思考过，如有这情况请选出一个你个人倾向的 答案。

\section{试题题目:}

1. 当一次重大考试就要来临时, 我总是在想别人比我聪明得多。
A. 是
B. 否

2. 如果我将要做一次智能测试，在做之前我会非常焦虑。
A. 是
B. 否

3. 如果我知道将会有一次智能测试, 在此之前我感到很自信, 很轻松。 

A. 是
B. 否

4. 参加重大考试时, 我会出很多汗。
A. 是
B. 否

5. 考试期间，我发现自己总是在想一些和考试内容无关的事。
A. 是
B. 否

6. 当一次 “突然袭击” 式的考试来到时, 我感到很怕。
A. 是
B. 否

7. 考试期间我经常想到会失败。
A. 是
B. 否

8. 重大考试后我经常感到紧张，以至胃不舒服。
A. 是
B. 否

9. 我对智能考试和期末考试之类的事总感到发怵。
A. 是
B. 否

10. 在一次考试中取得好成绩似乎并不能增加我在第二次考试中的信心。
A. 是
B. 否

11. 在重大考试期间我有时感到心跳很快。
A. 是
B. 否

12. 考试结束后我总是觉得可以比实际上做得更好。
A. 是
B. 否

13. 考试完毕后我总是感到很抑郁。
A. 是
B. 否

14. 每次期末考试之前, 我总有一种紧张不安的感觉。
A. 是
B. 否

15. 考试时，我的情绪反应不会干扰我考试。
A. 是
B. 否

16. 考试期间我经常很紧张, 以至本来知道的东西也忘了。
A. 是
B. 否

17. 复习重要的考试对我来说似乎是一个很大的挑战。
A. 是
B. 否 
18. 对某一门考试，我越努力复习越感到困惑。
A. 是
B. 否

19. 某门考试一结束，我试图停止有关担忧，但做不到。
A. 是
B. 否

20. 考试期间我有时会想我是否能完成学业。
A. 是
B. 否

21. 我宁愿写一篇论文，而不是参加一次考试，作为某门课程的成绩。
A. 是
B. 否

22. 我真希望考试不要那么烦人。
A. 是
B. 否

23. 我相信如果单独参加考试而且没有时间限制的话，我会考得更好。
A. 是
B. 否

24. 想着我在考试中能得多少分，影响了我的复习和考试。
A. 是
B. 否

25. 如果考试能废除的话，我想我能学得更好。
A. 是
B. 否

26. 我对考试抱这样的态度：虽然我现在不懂，但我并不担心。
A. 是
B. 否

27. 我真不明白为什么有些人对考试那么紧张。
A. 是
B. 否

28. 我很差劲的想法会干扰我在考试中的表现。
A. 是
B. 否

29. 我复习期末考试并不比复习平时考试更卖力。
A. 是
B. 否

30. 尽管我对某门考试复习很好，但我仍然感到焦虑。
A. 是
B. 否

31. 在重大考试前, 我吃不香。
A. 是
B. 否

32. 在重大考试前我发现我的手臂会颤抖。 

A. 是
B. 否

33. 在考试前我很少有 “临时抱佛脚” 的需要。
A. 是
B. 否

34. 校方应认识到有些学生对考试较为焦虑，而这会影响他们的考试成绩。
A. 是
B. 否

35. 我认为考试期间似乎不应该搞得那么紧张。
A. 是
B. 否

36. 一接触到发下的试卷, 我就觉得很不自在。
A. 是
B. 否

37. 我讨厌老师喜欢搞 “突然袭击”式的考试。
A. 是
B. 否 


\section{附件二：}

\section{自我效能感量表 GSES}

指导语：请仔细阅读每一道题，并根据自己实际情况进行作答。每一道题 都有 4 种答案（完全不正确、有点正确、多数正确、完全正确），请从 4 种答 案中选择一个适合你的, 在答题过程中不得漏题, 在同一题上不得煁酌太多时间, 根据看完题后的第一反应进行作答。有些题目可能与你不符或你从未思考过, 如有这情况请选出一个你个人倾向的答案。

\begin{tabular}{|c|l|c|c|c|c|}
\hline 题号 & \multicolumn{1}{|c|}{ 题项 } & $\begin{array}{c}\text { 完全 } \\
\text { 不正确 }\end{array}$ & $\begin{array}{l}\text { 正确 } \\
\text { 多数 } \\
\text { 正确完企 }\end{array}$ \\
\hline 1 & 如果我尽力去做的话, 我总是能够解决问题的 & & & & \\
\hline 2 & 即使别人反对我, 我仍有办法取得自己所要的 & & & & \\
\hline 3 & 对我来说, 坚持理想和达成目标是轻而易举的 & & & & \\
\hline 4 & 我自信能有效地应付任何突如其来的事情 & & & & \\
\hline 5 & 以我的才智, 我定能应付意料之外的情况 & & & \\
\hline 6 & 如果我付出必要的努力, 我一定能解决大多数的难题 & & & & \\
\hline 7 & 我能冷静地面对困难, 因为我相信自己处理问题的能力 & & & & \\
\hline 8 & 面对一个难题时, 我通常能找到几个解决方法 & & & & \\
\hline 9 & 有麻烦的时候, 我通常能想到一些应付的方法 & & & & \\
\hline 10 & 无论什么事情在我身上发生, 我都能应付自如 & & & & \\
\hline
\end{tabular}

